\title{
Updating superficial siderosis of the central nervous system: bleeding of a dorsal osteophyte into the subarachnoid space from a perforating artery
}

\author{
Carlo Brembilla, MD, ${ }^{1}$ Luigi Andrea Lanterna, PhD, ${ }^{1}$ Virginio Bonito, MD, ${ }^{2}$ \\ Margherita Gardinetti, MD, ${ }^{2}$ Gianluigi Dorelli, MD, ${ }^{1}$ Angela Dele Rampini, MD, ${ }^{1}$ Paolo Gritti, MD, ${ }^{3}$ \\ and Claudio Bernucci, MD1
}

Departments of ${ }^{1}$ Neurosurgery, ${ }^{2}$ Neurology, and ${ }^{3}$ Anaesthesia and Intensive Care, Pope John XXIII Hospital, Bergamo, Italy

\begin{abstract}
Superficial siderosis of the central nervous system (SSCNS) is an uncommon and often unrecognized disorder that results from recurrent and persistent bleeding into the subarachnoid space. Currently, there is no effective treatment for SSCNS. The identification and surgical resolution of the cause of bleeding remains the most reliable method of treatment, but the cause of bleeding is often not apparent. The identified sources of recurrent bleeding have typically included neoplasms, vascular malformations, brachial plexus or nerve root injury or avulsion, and previous head and spinal surgery. An association between recurrent bleeding in the CNS and dural abnormalities in the spine has recently been suggested. Dural tears have been identified in relation to a protruding disc or osteophyte. Also in these patients, the exact mechanism of bleeding remains unknown because of a lack of objective surgical data, even in patients who undergo neurosurgical procedures.
\end{abstract}

The present case concerns a 48-year-old man who presented with longstanding symptoms of mild hearing loss and mild gait ataxia. A diagnosis of SSCNS was made in light of the patient's history and the findings on physical examination, imaging, and laboratory testing. MRI and CT detected a small calcific osteophyte in the anterior epidural space of T8-9. The patient underwent surgical removal of the bone spur and dural tear repair. During the surgery, the authors detected a perforating artery, which was on the osteophyte, that was bleeding into the subarachnoid space. This case shows a possible mechanism of chronic bleeding from an osteophyte into the subarachnoid space. In the literature currently available, a perforating artery on an osteophyte bleeding into the subarachnoid space has never been described in SSCNS. https://thejns.org/doi/abs/10.3171/2018.7.SPINE18300

KEYWORDS superficial siderosis of the central nervous system; dural defect; epidural veins; spinal CSF leak; deferiprone

$\mathrm{S}$ UPERFICIAL siderosis of the central nervous system (SSCNS) is a progressive neurological syndrome that is characterized by chronic subarachnoid bleeding.? Currently, there is no effective treatment. As there is no definitive treatment, the identification and surgical resolution of the cause of bleeding remains the most reliable method. ${ }^{8}$ Recently, an association between recurrent bleeding in SSCNS and dural abnormalities in the spine has been suggested..$^{5}$ Dural tears have been identified in relation to a protruding disc or osteophyte, a disc-osteophyte complex, or calcification between the dura mater and arachnoid..$^{10}$ In most patients with SSCNS, the exact source of bleeding remains unknown because we lack ob- jective surgical data, even in patients who have undergone neurosurgical procedures.

\section{Case Report}

Presentation and Examination

This 48-year-old man presented with longstanding symptoms of mild hearing loss and mild gait ataxia. Three years earlier the patient, a professional runner, had reported a progressively unsteady gait and difficulties in coordinating his pace during physical activity. The patient came to the attention of Pope John XXIII Hospital in Bergamo, Italy, reporting a progressive worsening of the symptomatol-

ABBREVIATIONS SSCNS = superficial siderosis of the central nervous system.

SUBMITTED March 13, 2018. ACCEPTED July 10, 2018.

INCLUDE WHEN CITING Published online October 12, 2018; DOI: 10.3171/2018.7.SPINE18300. 

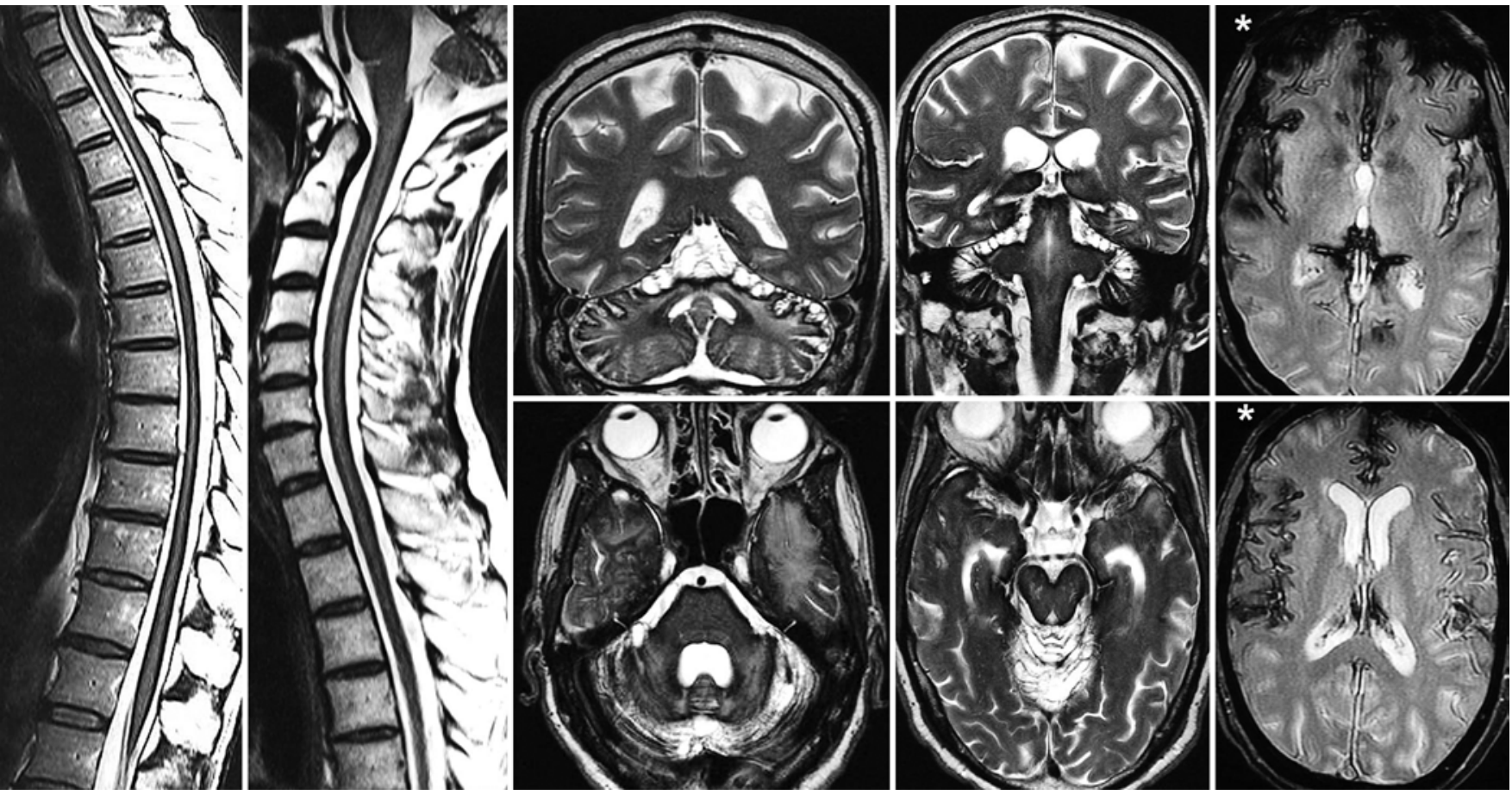

FIG. 1. Spinal cord and brain T2-weighted MRI scans showing hypointensity in the superficial areas of the CNS (asterisks in gradient-echo sequences), suggestive of hemosiderin deposition, also with a superior pattern of cerebellar atrophy.

ogy and with a cognitive deficit. Neurological examination revealed impaired olfactory sensation, severe sensorineural hearing loss, dysarthria, horizontal nystagmus, hyperactive tendon reflexes in all of his limbs, severe postural and gait ataxia, and mild long-term amnesia. A T2-weighted MRI study of the brain revealed hypointensity in the superficial areas of the CNS, which was suggestive of hemosiderin deposition, also with a superior pattern of cerebellar atrophy, a condition frequently associated with SSCNS (Fig. 1); no brain tumor or vascular lesion was reported. A lumbar puncture revealed a low opening pressure $\left(6 \mathrm{~cm} \mathrm{H}_{2} \mathrm{O}\right)$, xanthochromic cerebrospinal fluid (CSF) with the presence of red blood cells $(6000$ cells $/ \mathrm{ml})$, and a rise in protein range (ferritin $>200 \mathrm{ng} / \mathrm{ml}$ ). A diagnosis of SSCNS was made in light of the patient's history and the findings on physical examination, imaging, and laboratory testing.

The patient had no history of surgery or trauma in the CNS. His hemoglobin concentration and coagulation status were within reference ranges. To identify the bleeding source, he underwent angiography of the brain and the spinal cord, but the results were unremarkable. On critically reevaluating the spine MR images, a contrastenhanced spot in front of the T8-9 vertebral body was detected (Fig. 2), a finding compatible with the presence of an osteophyte. CT scanning confirmed the presence of a small calcific osteophyte in the anterior epidural space of T8-9 (Fig. 3). An association between recurrent bleeding in SSCNS and dural abnormalities in the spine has been suggested. ${ }^{5}$ Dural tears have been identified in relation to a protruding disc or osteophyte, a disc-osteophyte complex, or calcification between the dura and arachnoid..$^{10}$ After a lengthy period of establishing the diagnosis, this finding represented the only chance of identifying the cause of the subarachnoid bleeding and stopping the patient's progressive neurological syndrome.

\section{Operation}

Because of the clinical and radiological scenario, the patient underwent monitoring of somatosensory and motor evoked potentials, and a right T8-9 hemilaminectomy and arthrectomy. To explore the intradural and subarachnoidal space, a dorsal right paramedial durotomy was performed. On opening the dural sac, we saw evidence of chronic hypertrophic arachnoiditis. The fluid inside the arachnoid space was xanthochromic. In the arachnoid space, rising through an anterior dural wall defect, after denticulate ligament dissection, an osteophyte was detected. The bone spur showed bleeding from a perforating artery, which was likely the cause of the chronic subarachnoidal bleeding (Fig. 4). The bone lesion was removed and the bleeding stopped. After coagulation of the surrounding epidural space, where the epidural venous plexus started bleeding during surgical handling, the dural defect was sutured (Video 1).

VIDEO 1. Microscope video of the surgical operation (OPMI

Pentero 800, Carl Zeiss Surgical GmbH). Copyright Carlo Brembilla.

Published with permission. Click here to view.

Histological examination of the lesion revealed cartilaginous tissue and spongy bone tissue containing hematopoietic tissue.

\section{Postoperative Course}

After the operation, there were no changes in the pa- 


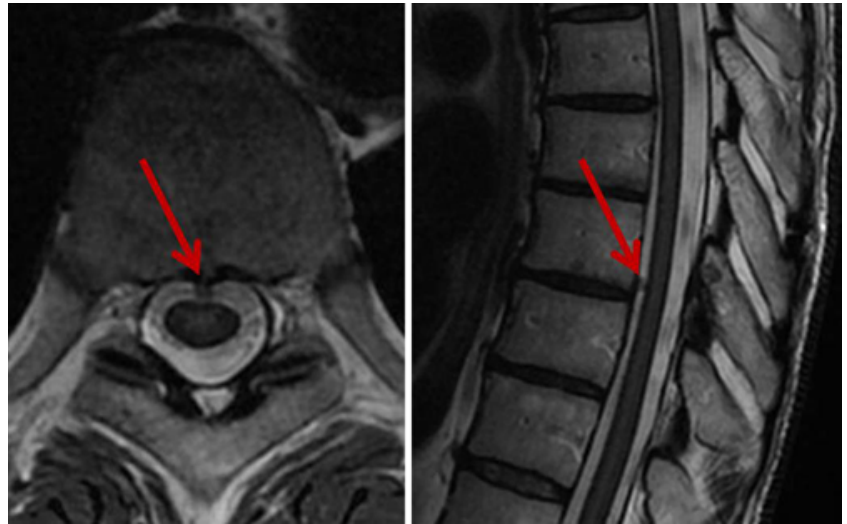

FIG. 2. MRI scans showing a contrast-enhanced spot in front of the T8-9 vertebral body (red arrow), a finding compatible with an osteophyte. Figure is available in color online only.

tient's neurological status, and he was started an iron chelator therapy with deferiprone (Ferriprox), with a dose of $30 \mathrm{mg} / \mathrm{kg} /$ day (about $2 \mathrm{~g} /$ day). The patient attended followup examinations at 1,3 , and 6 months, and then every 6 months thereafter, where he underwent head and spine MRI, lumbar punctures, and laboratory serum examination testing for any adverse effects of the iron chelator therapy. At 6 months after surgery, no traces of red blood cells were found in the CSF. At 2 years, MRI showed a considerable reduction in the CNS hemosiderin signal (Fig. 5); clinically the patient's cerebellar ataxia and his hearing impairment were slightly improved.

\section{Discussion}

SSCNS is an uncommon and often unrecognized disorder that results from hemosiderin deposition in the subpial layers of the CNS. The hemosiderin deposition arises from recurrent and persistent bleeding into the subarachnoid space?

The pathophysiology of SSCNS results primarily from an overproduction of hemoglobin degradation products, including free iron. ${ }^{4}$ The free iron from hemosiderin catalyzes the oxidation of hydrogen peroxide to superoxide, causing lipid peroxidation, membrane dysfunction, and ultimately cell death. Microscopically, the end result is neuronal loss, reactive gliosis, and axonal demyelination. ${ }^{5,6}$

Because of the microanatomy of the CNS and the specific CSF flow pattern, hemosiderin deposition generally occurs in the superior vermis, the crest of the cerebellar folia, the basal frontal lobe, temporal cortex, brainstem, spinal cord, nerve roots, and cranial nerves I and VIII. ${ }^{8}$ This may lead to progressive neurological deficits, such as sensorineural hearing loss, cerebellar ataxia, and signs of corticospinal tract dysfunction.

MRI is the investigating tool of choice. The typical MRI finding is a characteristic, marginal, confluent T2 hypointensity on the surface of the brain and spinal cord. This is better appreciated on gradient-echo sequences. Red blood cells or xanthochromia may be seen in CSF studies. ${ }^{6}$

Currently, there is no effective treatment for SSCNS.

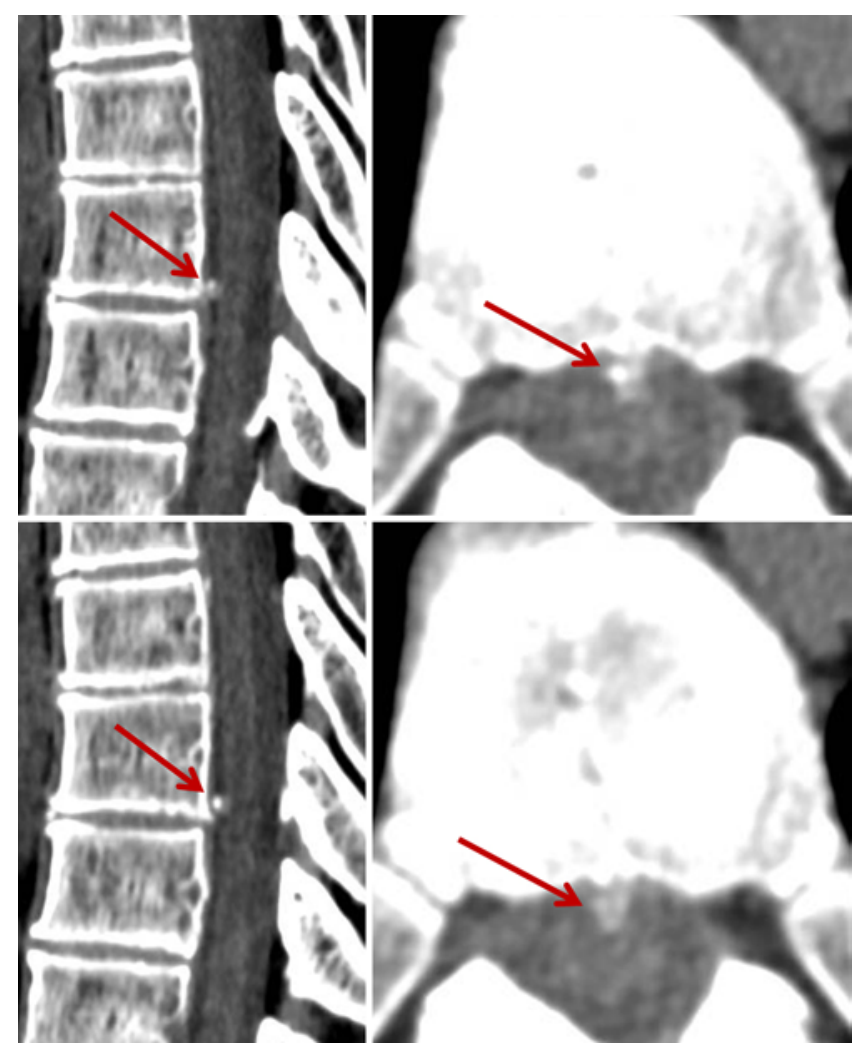

FIG. 3. CT scans confirming the presence of a small calcific osteophyte (red arrow) in the anterior epidural space of T8-9. Figure is available in color online only.

Medical management in the form of corticosteroids reportedly has been successful in a single case associated with anti-Ri antibodies. ${ }^{1,8}$ Penicillamine and large doses of vitamins $\mathrm{C}$ and $\mathrm{E}$ have also been effective in certain patients. ${ }^{1,8}$ Treatment with deferiprone, a lipid soluble iron chelator, has reduced hemosiderin deposition, as evidenced on MRI, in a cohort of 10 patients with SSCNS. ${ }^{9}$ However, prospectively designed efficacy studies are necessary to determine the clinical efficacy of deferiprone in SSCNS. As there is no definitive treatment for SSCNS, identification and surgical resolution of the bleeding source remains the most reliable method. Arresting the bleeding into the subarachnoid space seems to be accompanied by clinical stability or improvement that may sometimes be subjective. ${ }^{3,8,10}$

Despite extensive investigations (brain and spine MRI, CT myelography, MR angiography, cerebrospinal angiography), the cause of bleeding is often not apparent. The identified sources of recurrent bleeding in SSCNS have typically included neoplasms, vascular malformations, brachial plexus or nerve root injury or avulsion, and previous head and spinal surgery. ${ }^{2}$

Recently, an association between recurrent bleeding in SSCNS and dural abnormalities in the spine has been suggested. ${ }^{5,10}$ Dural tears have been identified in relation to a protruding disc or osteophyte, a disc-osteophyte complex, or calcification between the dura and arachnoid. A dynamic CT myelogram and a digital subtraction myelo- 
Brembilla et al.
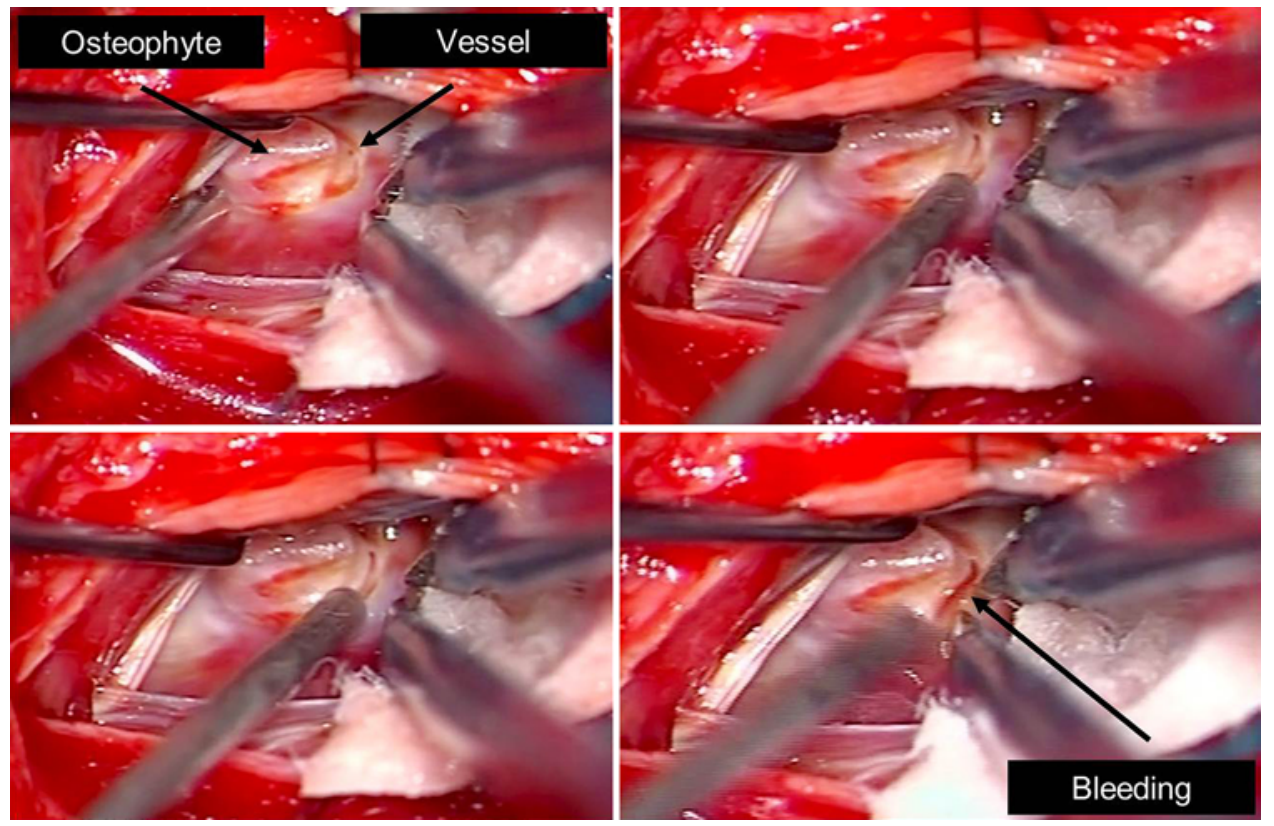

FIG. 4. The perforating artery bleeding on the bone spur is likely the cause of the subarachnoid chronic bleeding. Figure is available in color online only.

gram could be helpful in identifying dural defects, thereby determining a potential site for surgery. ${ }^{6}$ On occasion, a longitudinally fluid-filled collection in the spinal canal has been observed on MRI. The predominantly ventral location of the fluid-filled collections seems to confirm the degenerative disc disease pathogenesis. Two theories have been proposed for the source of bleeding in patients with SSCNS associated with a ventral spinal CSF leak. The first theory is that brain sagging due to intracranial hypotension may lead to bleeding from bridging veins on
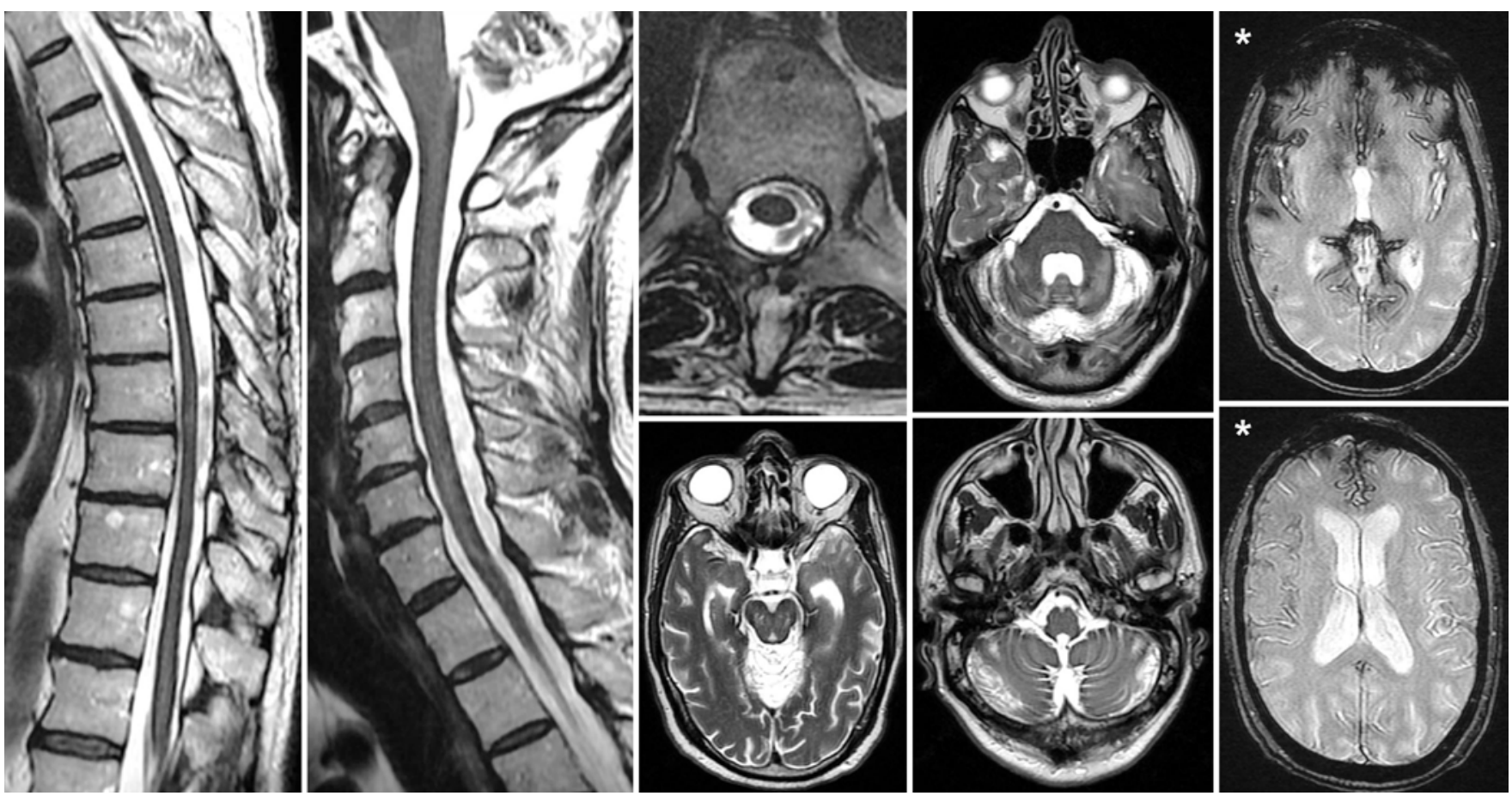

FIG. 5. Two-year postoperative T2-weighted MRI studies of the spinal cord and brain showing a considerable reduction in the CNS hemosiderin signal (asterisks in gradient-echo sequences). 
the surface of the cerebellum, ${ }^{5}$ and the second theory is that the bleeding source may be fragile vessels around the dural defect. However, the exact bleeding source remains unknown because of a lack of objective data, even in patients who have undergone neurosurgery. 5,10

This case shows a possible mechanism of chronic bleeding from an osteophyte into the subarachnoid space. In the currently available literature, a perforating artery on an osteophyte bleeding into the subarachnoid space has not been described. As in other cases, in our patient the vessels in the epidural space around the dural defect were very fragile; their involvement in the chronic subarachnoid bleeding cannot be ruled out. Despite the fact that in this case the cause of bleeding was identified and excluded and the postoperative neuroradiological images and CSF examinations were refined, the patient's neurological status was only slightly improved. This was probably because of his longstanding symptoms and, consequently, the longstanding effects of hemoglobin degradation products on the CNS.

The featured case is only observational. More cases must be observed to confirm the mechanisms of subarachnoid bleeding from vertebral osteophytes related to dural tears. Another issue that should be elaborated on is how extensive the search should be for spinal causes of hemosiderosis. A longitudinally fluid-filled collection in the spinal canal can sometimes be observed with MRI, and this finding could identify a potential site for surgery. A dynamic CT myelogram and a digital subtraction myelogram could also be helpful in identifying dural defects. ${ }^{6}$ However, in the present case, as in the current literature, no evidence could be found regarding this issue.

Despite different sources of bleeding in the presence of a vertebral osteophyte and dural tears, the solution appears to be the same: osteophyte removal and dural tear closure.

\section{References}

1. Angstwurm K, Schielke E, Zimmer C, Kivelitz D, Weber JR: Superficial siderosis of the central nervous system: response to steroid therapy. J Neurol 249:1223-1225, 2002

2. Bonito V, Agostinis C, Ferraresi S, Defanti CA: Superficial siderosis of the central nervous system after brachial plexus injury. Case report. J Neurosurg 80:931-934, 1994

3. Egawa S, Yoshii T, Sakaki K, Inose H, Kato T, Kawabata S, et al: Dural closure for the treatment of superficial siderosis. J Neurosurg Spine 18:388-393, 2013
4. Koeppen AH, Dickson AC, Chu RC, Thach RE: The pathogenesis of superficial siderosis of the central nervous system. Ann Neurol 34:646-653, 1993

5. Kumar N: Beyond superficial siderosis: introducing "duropathies." Neurology 78:1992-1999, 2012

6. Kumar N: Neuroimaging in superficial siderosis: an in-depth look. AJNR Am J Neuroradiol 31:5-14, 2010

7. Kumar N, Cohen-Gadol AA, Wright RA, Miller GM, Piepgras DG, Ahlskog JE: Superficial siderosis. Neurology 66:1144-1152, 2006

8. Leussink VI, Flachenecker P, Brechtelsbauer D, Bendszus M, Sliwka U, Gold R, et al: Superficial siderosis of the central nervous system: pathogenetic heterogeneity and therapeutic approaches. Acta Neurol Scand 107:54-61, 2003

9. Levy M, Llinas R: Pilot safety trial of deferiprone in 10 subjects with superficial siderosis. Stroke 43:120-124, 2012

10. Takai K, Komori T, Niimura M, Taniguchi M: Superficial siderosis of the central nervous system associated with intraspinal hemorrhage from ventral thoracic epidural veins and a ventral spinal CSF leak: case report. J Neurosurg Spine 26:751-753, 2017

\section{Disclosures}

The authors report no conflict of interest concerning the materials or methods used in this study or the findings specified in this paper.

\section{Author Contributions}

Conception and design: Brembilla, Lanterna, Bonito, Bernucci. Acquisition of data: Bonito, Gardinetti, Dorelli, Rampini. Analysis and interpretation of data: Bonito. Drafting the article: Brembilla. Critically revising the article: Brembilla, Lanterna, Gritti, Bernucci. Reviewed submitted version of manuscript: all authors. Approved the final version of the manuscript on behalf of all authors: Brembilla. Administrative/technical/material support: Gardinetti, Dorelli, Rampini. Study supervision: Brembilla.

\section{Supplemental Information Videos \\ Video 1. https://vimeo.com/280529007.}

\section{Correspondence}

Carlo Brembilla: Pope John XXIII Hospital, Bergamo, Italy. carlinobrembo@hotmail.com; carlinobrembo@gmail.com. 